\title{
Current treatment of T1N0 squamous cell carcinoma of the glottic larynx
}

\author{
William M. Mendenhall $\cdot$ Robert P. Takes $\cdot$ Jatin P. Shah $\cdot$ Patrick J. Bradley • \\ Jonathan J. Beitler · Primož Strojan • Carlos Suárez • Juan P. Rodrigo • \\ Nabil F. Saba $\cdot$ Alessandra Rinaldo $\cdot$ Jochen A. Werner $\cdot$ Alfio Ferlito
}

Received: 17 October 2014/ Accepted: 30 October 2014/Published online: 8 November 2014

(c) Springer-Verlag Berlin Heidelberg 2014

The optimal treatment of patients with T1N0 squamous cell carcinoma (SCC) of the glottic larynx remains controversial [1-3].

T1 glottic SCC as defined by the AJCC/UICC system [4] is "Tumor limited to the vocal cords (which may involve the anterior or posterior commissure) with normal mobility". This disease stage is further subdivided into T1a: tumor limited to one vocal cord, and T1b: tumor involving both vocal cords. Accurate assessment of the true $\mathrm{T}$ stage of glottic SCC (disease invasion of contiguous tissues) requires careful clinical, endoscopic and

This article was written by members of the International Head and Neck Scientific Group (http://www.IHNSG.com).

W. M. Mendenhall

Department of Radiation Oncology, University of Florida, Gainesville, FL, USA

R. P. Takes

Department of Otolaryngology-Head and Neck Surgery,

Radboud University Medical Center, Nijmegen, The Netherlands

J. P. Shah

Head and Neck Service, Memorial Sloan-Kettering Cancer

Center, New York, NY, USA

P. J. Bradley

Department of Otolaryngology-Head and Neck Surgery,

Nottingham University Hospitals, Queens Medical Centre

Campus, Nottingham, UK

\section{J. J. Beitler}

Departments of Radiation Oncology, Otolaryngology and Medical Oncology, The Winship Cancer Institute of Emory University, Atlanta, GA, USA

P. Strojan

Department of Radiation Oncology, Institute of Oncology,

Ljubljana, Slovenia stroboscopic examination. Radiological imaging methods to determine the extent of disease is usually not needed in T1 glottic SCC except for tumors at the anterior commissure [5]. The use of functional assessment of voice, both pre-treatment and again at an agreed upon post-treatment time (12 months) may be very useful in evaluating vocal function. However, no standard test of voice quality has been universally accepted although standardized protocols for functional assessment have been suggested [6].

Treatment options for T1 glottic SCC include radiotherapy (RT), transoral laser resection (TLR), and open partial laryngectomy [7-10]. Voice quality after open partial laryngectomy is significantly worse compared to RT and TLR so that this alternative is rarely employed. The

\section{Suárez · J. P. Rodrigo}

Department of Otolaryngology, Hospital Universitario Central de Asturias, Oviedo, Spain

C. Suárez · J. P. Rodrigo

Instituto Universitario de Oncología del Principado de Asturias, Oviedo, Spain

\section{N. F. Saba}

Department of Hematology and Medical Oncology, The Winship Cancer Institute of Emory University, Atlanta, GA, USA

A. Rinaldo · A. Ferlito $(\square)$

University of Udine School of Medicine, Piazzale S. Maria della Misericordia, 33100 Udine, Italy

e-mail: a.ferlito@uniud.it

\section{J. A. Werner}

Department of Otolaryngology-Head and Neck Surgery, Philipp University, Marburg, Germany 
role of chemotherapy alone in early stage laryngeal cancer (including glottis) remains investigational $[11,12]$.

The decision whether to select RT or TLR for the treatment of T1 glottic SCC depends on a number of factors, including the location and extent of the tumor, the medical condition of the patient, the likelihood of tumor control after treatment, anticipated functional outcome (voice quality), the expertise of the attending physicians and logistical considerations. In this complex decisionmaking process, we should also include patient preference, after an informed discussion of the pros and cons of each treatment modality.

Controversies regarding the best option for treatment exist because of the lack of high-quality prospective randomized trials comparing these modalities of treatment $[3$, 13]. Both treatment options are characterized differently. TLR can be done as a single procedure on an outpatient basis, whereas RT is delivered once daily on weekdays over 5-7 weeks. The likelihood of local control after RT or TLR is equivalent and is approximately $85-95 \%$ [1-3]. However, in the reporting of local control after TLR, there is a difference between local control after the first procedure and "ultimate" local control after a subsequent TLR. In interpreting these data, one needs to understand that selection bias may exist in choosing the treatment employed. An advantage of RT is that it is applicable to all patients with T1N0 SCCs. TLR is usually applied in lessadvanced tumors as most physicians keep in mind the fact that the more of the glottis that is involved with SCC, requiring a wider resection, the poorer the voice quality after resection $[8,14]$. Thus it is likely that the more favorable cases are included in the reports on outcomes from TLR.

In some patients with very limited midcord lesions, the biopsy taken for diagnostic purposes may in fact have completely removed the small and superficial tumor; a fact demonstrated if followed by TLR but remaining in question if followed by radiotherapy. However, the absence of tumor in the re-excision specimen implies that it has either been completely removed by the biopsy or is not found (missed) by the pathologist. The only way to know whether a diagnostic biopsy has completely removed the tumor is to serially section the biopsy specimen for margins and administer no additional treatment, either TLR or RT.

Voice quality is reported to be comparable for both treatment modalities but again a selection bias may have been introduced in retrospective analyses [5, 8, 15], and although both TLR and radiotherapy have been found to offer similar objective measurement results, it seems that, from the patient's perception, there is a reduced impact on voice quality after RT $[16,17]$. Another advantage of TLR is that it can be repeated several times in contrast to RT. The ability to repeat TLR may contribute to the fact that the likelihood of laryngeal preservation may be higher when TLR can be offered as initial treatment [3, 5]. Many patients with recurrences after RT will undergo total laryngectomy. However, laryngeal preservation may be feasible with salvage open partial laryngectomy or TLR in selected patients after radiation failure [18-20]. About onethird of such recurrent cancers are suitable for conservation surgery [21]. Patients with anterior commissure involvement will provide technical challenges and, even in experienced hands, may have local control rates that are somewhat lower compared with T1 SCCs without anterior commissure invasion [5, 22]. Efficacy of RT is not affected by involvement of the anterior commissure. However, voice quality is likely to be worse after TLR in these cases. Patients with significant medical co-morbidities who are poor candidates for anesthesia may be better treated with RT. On the other hand, some frail elderly patients may prefer the short procedure of a TLR over a full course of radiotherapy. Another important point to be taken into consideration is that in several studies TLR appeared to be the most cost-effective treatment of early glottic SCC, radiation therapy being two- to fourfold more expensive $[15,23]$. The presence and extent of a cost differential will vary with the medical system.

The selection of patients for either treatment modality depends on all of the above-mentioned factors and this may also explain the lack of prospective randomized trials comparing both modalities. To randomize patients with T1 glottic SCC between RT and TLR without consideration of the above-mentioned factors such as, e.g. extent and depth of invasion of the tumor (rather than $\mathrm{T}$ classification as such), occupation and social context of the patient, patient preferences, comorbidity, etc., may be considered unethical and will make the design of a prospective study very difficult. Attempts at conducting such a trial have met with significant difficulties in accrual, and it has been deemed to be nearly impossible to conduct such a trial [13].

The only prospective comparison of RT and TLR for T1aN0 glottic SCC reported so far included only 56 patients over a 10-year period [24]. At 24 months posttherapy, a more breathy voice and wider glottis gap were found after TLR. Also, irradiated patients reported less hoarseness-related inconvenience in daily living, although overall voice quality and local control were similar between the two groups. Small patient numbers not allowing identification of eventual further differences between the treatments, short follow-up and absence of information on important voice quality modifying parameters (e.g. smoking habits, also having an adverse effect during RT) preclude firm conclusions. It seems, however, that RT may be preferred treatment option for patients with more demanding requirements for voice quality. 
In case RT is the selected treatment modality, a successful fractionation schedule for $\mathrm{T} 1$ glottic cancer is 63 Gy in 28 once-daily fractions [25, 26]. Despite the very low probability of a significant complication after this treatment, many radiation oncologists prefer a more protracted schedule [25]. The reimbursement schedule in some countries, including the United States, increases with the number of fractions (treatments) thus creating a potential conflict of interest. For whatever reason, many radiation oncologists (in North America and elsewhere) select a commonly employed fractionation schedule that consists of $66 \mathrm{~Gy}$ in 33 fractions, which produces a significantly inferior result $[25,26]$. Recently, the American College of Radiology (ACR) Expert Panel on Radiation OncologyHead and Neck Cancer developed consensus recommendations for treatment of T1 glottic SCC. They concluded that "Treatment planning is complex and decisions nuanced". And "Best treatment for a particular cancer cannot be defined without consideration of the lesion's location, extent, depth of invasion, and quality of surgical exposure during direct laryngoscopy" [26]. But regardless of the modality chosen, physicians should track their own patient's functional and disease-free survival data rather than rely on the best reported (published) results from the most experienced institutions. Analysis of outcomes should include tumor control, survival, functional outcomes (quality of voice) and larynx preservation rates.

In conclusion, the choice between treatment modalities for early stage glottic SCC should be based on careful considerations of all the factors discussed above, and made by the clinician and the patient. For optimal decisionmaking, the anticipated oncological and functional outcome from each treatment strategy should be considered. It is important to bear in mind that any physician or a specialist treatment center for early glottic SCC, is aware of their own outcome results.

\section{References}

1. Mendenhall WM, Werning JW, Hinerman RW, Amdur RJ, Villaret DB (2004) Management of T1-T2 glottic carcinomas. Cancer 100:1786-1792

2. Chera BS, Amdur RJ, Morris CG, Kirwan JM, Mendenhall WM (2010) T1N0 to T2N0 squamous cell carcinoma of the glottic larynx treated with definitive radiotherapy. Int J Radiat Oncol Biol Phys 78:461-466

3. Yoo J, Lacchetti C, Hammond JA, Gilbert RW (2013) Role of endolaryngeal surgery (with or without laser) versus radiotherapy in the management of early (T1) glottic cancer: a systematic review. Head Neck. doi:10.1002/hed.23504

4. Sobin LH, Wittekind C (2010) UICC TNM classification of malignant tumours. Wiley-Liss, New York

5. Bradley PJ, Rinaldo A, Suárez C, Shaha AR, Leemans CR, Langendijk JA, Patel SG, Ferlito A (2006) Primary treatment of the anterior vocal commissure squamous carcinoma. Eur Arch Otorhinolaryngol 263:879-888

6. Dejonckere PH, Bradley P, Clemente P, Cornut G, CrevierBuchman L, Friedrich G, Van De Heyning P, Remacle M, Woisard V; Committee on Phoniatrics of the European Laryngological Society (ELS) (2001) A basic protocol for functional assessment of voice pathology, especially for investigating the efficacy of (phonosurgical) treatments and evaluating new assessment techniques. Guideline elaborated by the Committee on Phoniatrics of the European Laryngological Society (ELS). Eur Arch Otorhinolaryngol 258:77-82

7. Remmelts AJ, Hoebers FJ, Klop WM, Balm AJ, Hamming-Vrieze O, van den Brekel MW (2013) Evaluation of lasersurgery and radiotherapy as treatment modalities in early stage laryngeal carcinoma: tumour outcome and quality of voice. Eur Arch Otorhinolaryngol 270:2079-2087

8. Taylor SM, Kerr P, Fung K, Aneeshkumar MK, Wilke D, Jiang Y, Scott J, Phillips J, Hart RD, Trites JR, Rigby MH (2013) Treatment of T1b glottic SCC: laser vs. radiation-a Canadian multicenter study. J Otolaryngol Head Neck Surg 42:22

9. Al-Mamgani A, van Rooij PH, Woutersen DP, Mehilal R, Tans L, Monserez D, Baatenburg de Jong RJ (2013) Radiotherapy for T1-2N0 glottic cancer: a multivariate analysis of predictive factors for the long-term outcome in 1050 patients and a prospective assessment of quality of life and voice handicap index in a subset of 233 patients. Clin Otolaryngol 38:306-312

10. Laoufi S, Mirghani H, Janot F, Hartl DM (2014) Voice quality after treatment of T1a glottic cancer. Laryngoscope 124:1398-1401

11. Holsinger FC, Lin HY, Bassot V, Laccourreye O (2009) Platinbased exclusive chemotherapy for selected patients with squamous cell carcinoma of the larynx and pharynx. Cancer 115:3909-3918

12. Mendenhall WM, Tannehill SP (1999) Should chemotherapy alone be the initial treatment for glottic squamous cell carcinoma? Eur J Cancer 35:1309-1310

13. Hamilton DW, de Salis I, Donovan JL, Birchall M (2013) The recruitment of patients to trials in head and neck cancer: a qualitative study of the EaStER trial of treatments for early laryngeal cancer. Eur Arch Otorhinolaryngol 270:2333-2337

14. Mendelsohn AH, Xuan Y, Zhang Z (2014) Voice outcomes following laser cordectomy for early glottic cancer: a physical model investigation. Laryngoscope 124:1882-1886

15. Goor KM, Peeters AJ, Mahieu HF, Langendijk JA, Leemans CR, Verdonck-de Leeuw IM, van Agthoven M (2007) Cordectomy by $\mathrm{CO}_{2}$ laser or radiotherapy for small T1a glottic carcinomas: costs, local control, survival, quality of life, and voice quality. Head Neck 29:128-136

16. Núñez Batalla MJ, Caminero Cueva B, Señaris González JL, Llorente Pendás C, Gorriz Gil A, López Llames R, Alonso Pantiga C, Nieto S (2008) Voice quality after endoscopic laser surgery and radiotherapy for early glottic cancer: objective measurements emphasizing the Voice Handicap Index. Eur Arch Otorhinolaryngol 265:543-548

17. Suárez C, Rodrigo JP, Silver CE, Hartl DM, Takes RP, Rinaldo A, Strojan P, Ferlito A (2012) Laser surgery for early to moderately advanced glottic, supraglottic and hypopharyngeal cancers. Head Neck 34:1028-1035

18. Ganly I, Patel SG, Matsuo J, Singh B, Kraus DH, Boyle JO, Wong RJ, Shaha AR, Lee N, Shah JP (2006) Results of surgical salvage after failure of definitive radiation therapy for early-stage squamous cell carcinoma of the glottic larynx. Arch Otolaryngol Head Neck Surg 132:59-66

19. Sewnaik A, van den Brink JL, Wieringa MH, Meeuwis CA, Kerrebijn JD (2005) Surgery for recurrent laryngeal carcinoma after radiotherapy: partial laryngectomy or total laryngectomy for a better quality of life? Otolaryngol Head Neck Surg 132:95-98 
20. Ramakrishnan Y, Drinnan M, Kwong FN, Grant DG, Mehanna H, Jones T, Paleri V (2014) Oncologic outcomes of transoral laser microsurgery for radiorecurrent laryngeal carcinoma: a systematic review and meta-analysis of English-language literature. Head Neck 36:280-285

21. Agra IM, Ferlito A, Takes RP, Silver CE, Olsen KD, Stoeckli SJ, Strojan P, Rodrigo JP, Gonçalves Filho J, Genden EM, Haigentz M Jr, Khafif A, Weber RS, Zbären P, Suárez C, Hartl DM, Rinaldo A, Kim KH, Kowalski LP (2012) Diagnosis and treatment of recurrent laryngeal cancer following initial nonsurgical therapy. Head Neck 34:727-735

22. Rodel RM, Steiner W, Muller RM, Kron M, Matthias C (2009) Endoscopic laser surgery of early glottic cancer: involvement of the anterior commissure. Head Neck 31:583-592

23. Phillips TJ, Sader C, Brown T, Bullock M, Wilke D, Trites JR, Hart R, Murphy M, Taylor SM (2009) Transoral laser microsurgery versus radiation therapy for early glottic cancer in Canada: cost analysis. J Otolaryngol Head Neck Surg 38:619-623
24. Aaltonen LM, Rautiainen N, Sellman J, Saarilahti K, Mäkitie A, Rihkanen H, Laranne J, Kleemola L, Wigren T, Sala E, Lindholm P, Grenman R, Joensuu H (2014) Voice quality after treatment of early vocal cord cancer: a randomized trial comparing laser surgery with radiation therapy. Int $\mathbf{J}$ Radiat Oncol Biol Phys 90:255-260

25. Yamazaki H, Nishiyama K, Tanaka E, Koizumi M, Chatani M (2006) Radiotherapy for early glottic carcinoma (T1N0M0): results of prospective randomized study of radiation fraction size and overall treatment time. Int $\mathbf{J}$ Radiat Oncol Biol Phys 64:77-82

26. Ridge JA, Lawson J, Yom SS, Garg MK, McDonald MW, Quon H, Saba N, Salama JK, Smith RV, Worden F, Yeung AR, Beitler JJ (2014) American College of Radiology Appropriateness Criteria $\left({ }^{\circledR}\right)$ treatment of stage I T1 glottic cancer. Head Neck 36:3-8 